\title{
HISTORIA
}

\section{LOS CUIDADOS PRESTADOS POR LAS MATRONAS EN EL BIERZO ALTO (León): CIEN AÑOS DE EVOLUCIÓN}

\author{
Elena Andina Díaz.
}

Diplomada en Enfermería y Lda. en Enfermería (Bachelor of Nursing).

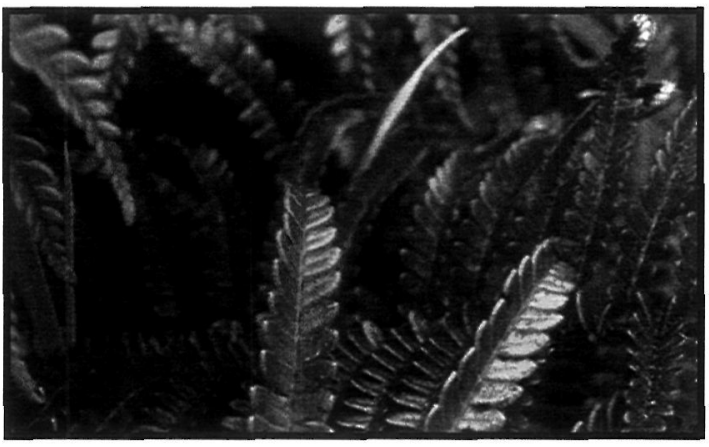

\section{CARE GIVEN BY MIDWIVES IN BIERZO ALTO (LEON, SPAIN): A HUNDRED YEARS EVOLUTION}

ก The role played by midwives throughout history has been intimately conditioned by the culture of the society in which they lived: habits, beliefs and rites. The aim of the present work is approaching to knowledge and evolution of care given by midwives during the XX century in this Spanish area, Bierzo Alto, located in Leon.

Data from various literature sources were analysed, specially from "El Ciclo Vital en España. Encuesta del Ateneo de Madrid, 1901-1902" and "Anuario General de España Bailly-BailliereRiera, 1930 and 1944". As complementary information, a field work was carried out in which -thanks to the data provided by "people who knew"- the role played by midwives could be followed and compared in three different moments: 1901, 1950 and 2002.

Finally, this article is aimed to contribute if only modestly to "Antropology of Nursing", deepening into culture of care during the first stage of the vital cycle.
Key words: Anthropology of Nursing, Midwives, History, Culture of Care.

\section{RESUMEN}

El papel que las matronas han desempeñado a lo largo de la historia ha estado íntimamente condicionado por la cultura de la sociedad en la que vivían: las costumbres, las creencias y los ritos. El objeto del presente trabajo fue acercarse al conocimiento y evolución de los cuidados brindados por las matronas a lo largo del siglo XX en una zona concreta de la geografía española, la subcomarca del Bierzo Alto, ubicada en la provincia de León.

Nos basamos para ello en el análisis de datos extraídos de diferentes fuentes bibliográficas en los que aparece información sobre el tema, principalmente en la obra "El Ciclo Vital en España. Encuesta del Ateneo de Madrid, 1901-1902", y en el "Anuario General de España Bailly-BaillièreRiera", años 1930 y 1944. Como complemento de esta información escrita, se realizó un "trabajo de campo", en el que gracias a los datos facilitados por "personas conocedoras" pudimos comparar y seguir la función realizada por el colectivo de matronas y parteras en tres momentos diferentes: 1901,1950 y 2002.

Finalmente añadir que con este pequeño estudio se pretende hacer una nueva aportación al campo de la "Antropología de la Enfermería", lo cual contribuirá, aunque sea de forma modesta, a profundizar un poco más en la cultura de los cuidados referida a la primera etapa del ciclo vital.

Palabras clave: antropología de la enfermería, matronas, historia, cultura de los cuidados. 


\section{INTRODUCCIÓN}

\subsection{ANTECEDENTES Y ESTADO ACTUAL DEL TEMA}

Desde el origen de la humanidad, los cuidados que sustentan la vida: la lactancia, la crianza, cuidados de los enfermos, alimentación o higiene, han estado vinculados a la figura de la mujer. Ella ha sido la encargada de proporcionar esa atención continuada a la familia, asegurando que se cubrieran las necesidades básicas del hogar. Este "rol biológico" en el que se incluyen las actividades desarrolladas por las mujeres en función de su capacidad biológica, basaba sus conocimientos en el empirismo principalmente, y se trasmitía de generación en generación (Siles González, 1996).

El objetivo planteado en el presente trabajo consiste en estudiar y reflejar la evolución de uno de los cuidados indiscutiblemente enfermeros atribuidos desde el principio de los tiempos a la mujer, la atención en el embarazo, parto y puerperio.

Remontándonos a nuestros orígenes, ya en la Biblia se hace mención al trabajo de la partera, mujer que se ocupaba de asistir los partos, y que gozaba de gran prestigio social (García Martínez y García Martínez, 1999). Durante muchos siglos el cuidado administrado por las mujeres en el momento del parto no fue considerado una verdadera profesión, sólo un rol femenino. Es a partir del siglo XV cuando aparecen las primeras normativas legales sobre cuidados de parteras y comadronas; ya en el siglo XVIII, diferentes autores publican libros sobre el tema, y algunas instituciones proponen medidas para instruir a las matronas (Cuadri Duque, 1999). Pero no será hasta finales del siglo XIX cuando las matronas, junto con las maestras, se conviertan en las primeras mujeres en España que logren que sus actividades paradomésticas tengan el reconocimiento de profesiones regladas (Siles González, 1995; Siles González, 1996).

Basándonos en el análisis Post-Oc de diferentes documentos escritos existentes sobre el tema (Siles González, 2001), y utilizando como complemento la información oral obtenida tras un "trabajo de campo" realizado a tal fin, se fija como objetivo conocer la evolución durante el siglo XX de los cuidados administrados por las matronas en una zona concreta de la geografía española, la subco- marca del Bierzo Alto, perteneciente a la provincia de León. Se trata, evidentemente, de una primera aproximación, abierta a otras posteriores, para recoger información sobre las actividades habituales desarrolladas por este colectivo. Y también de aportar un nuevo grano de arena al campo de la "Antropología de la Enfermería" (Littlewood y Holden, 1991), que contribuya a definir los rasgos socioculturales de esta profesión, sus prácticas, tradiciones y rituales (Mulhall, 1996).

\subsection{HIPÓTESIS DE TRABAJO}

- La atención de Enfermería en el embarazo y parto está basada hoy en día en el paradigma biomédico dominante, obviando ese lado mágico y religioso que acompañaba hace un siglo a la atención prestada por las antiguas matronas.

\subsection{OBJETIVOS}

- Constatar el papel desempeñado por las matronas, en el Bierzo Alto, en el año 1901.

- Constatar el papel desempeñado por las matronas, en dicha zona, a mediados del siglo XX.

- Constatar el papel desempeñado por las matronas, en dicha zona, en el año 2002.

- Conocer el número de matronas que ejercían y ejercen en la zona a estudio en los períodos de tiempo citados.

\subsection{EQUIPAMIENTO MÉDICO E INFRAES- TRUCTURA SANITARIA DEL BIERZO ALTO}

El Bierzo Alto ocupa la parte oriental de la comarca del Bierzo, la más extensa, variada y mejor delimitada de la provincia de León, ocupando una extensión es de $627 \mathrm{Km}^{2}$. Bembibre se ha consolidado como la capital administrativa, comercial y de servicios de la subcomarca, y también como la segunda ciudad del Bierzo después de Ponferrada.

Desde el punto de vista de la asistencia sanitaria, no conocemos con detalle cuál era el equipamiento y el grado de cobertura existente en la zona a principios del siglo XX. En 1930, cuando la población de la cuenca ascendía a 17.193 habitantes, había para toda la cuenca un total 11 médicos ( 5 de ellos en Bembibre, siendo 1 además ginecólogo); 3 farmacias (2 en Bembibre); 1 practicante y 2 comadronas ( 1 en Bembibre) desconociéndose si 
eran o no tituladas (Anuario Bailly-Baillière, 1930). Cabe comentar al respecto que aunque el índice de facultativos era, para aquel momento considerable, existía el inconveniente de las pésimas comunicaciones, sobre todo en los núcleos montañosos que ocupan buena parte del territorio, por lo que, muchas veces, antes de acudir al médico con los enfermos se agotaban todos los recursos de la medicina popular, con el riesgo de que cuando finalmente se solicitaban sus servicios, éste llegase tarde.

Años después de finalizada la contienda civil, en 1945 , la situación había empeorado sensiblemente al situarse en 8 el número de médicos (4 en Bembibre, 1 de ellos ginecólogo); 2 farmacias en Bembibre; 1 practicante y 4 comadronas (1 en Bembibre) sin que conozcamos tampoco si eran o no tituladas (Anuario Bailly-Baillière, 1945).

Durante la década de los sesenta y setenta, y a pesar del importante crecimiento demográfico derivado de la inmigración, al tratarse de una comarca minera en auge; la atención médica continuaba prestándose en los consultorios particulares de los facultativos, hasta que, ya a finales de los setenta, se abrió el primer Ambulatorio de la Seguridad Social en Bembibre, cuya capacidad se vio desbordada de inmediato. Por fin, en 1988 se inauguró el nuevo Centro de Salud de Bembibre que dispone de consultas de medicina general, pediatría, odontología, enfermería y matrona. Además, durante la década de los noventa, buena parte de las cabeceras municipales y pueblos más reivindicativos han ido consiguiendo su pequeño consultorio médico (Andina Yanes, trabajo en prensa).

La actual Zona Básica de Salud de Bembibre, que coincide básicamente con los municipios que integran el Bierzo Alto: Bembibre, Castropodame, Folgoso de la Ribera, Igüeña, Noceda, Torre del Bierzo; y además las entidades menores de La Silva y Montealegre, pertenecientes al ayuntamiento de Villagatón (quedando fuera de la Zona el municipio de Congosto que sí pertenece a la Cuenca del Boeza). Cuenta con un Centro de Salud situado en Bembibre y 37 consultorios locales repartidos por los 47 pueblos de la subcomarca. La población cubierta ascendía, a principios del año 2002, a 19.499 habitantes. Para atender a las muje- res embarazadas de la Zona existe una matrona que ejerce sus funciones en el Centro de Salud de Bembibre (Datos obtenidos de la Gerencia de Atención Primaria del Área de Salud del Bierzo, 2002).

En cuanto a la Atención Especializada, el equipamiento hospitalario más cercano está en Ponferrada, distante unos 20 kilómetros de la cabecera de la subcomarca. Aunque anteriormente había en dicha ciudad varias clínicas privadas, al tratarse de centros de pago sólo eran utilizadas en casos extremos o por pacientes de un nivel económico alto. Así pues, hasta la apertura, el año 1968, de la Residencia de la Seguridad Social de Ponferrada (actual Hospital del Bierzo), el lugar de atención al parto había sido tradicionalmente la casa de la gestante o de la familia (generalmente de la madre de ésta), e incluso después de esa fecha se continuaron realizando, durante un tiempo, partos domiciliarios, especialmente en los pueblos más apartados y en familias de menor nivel cultural.

Actualmente podemos decir que la atención hospitalaria al parto se ha universalizado, bien en el Hospital del Bierzo, ya citado, o en las clínicas privadas.

\section{MATERIAL Y MÉTODO}

Con el presente trabajo se pretendió pues realizar un análisis del papel desempeñado por las matronas que ejercieron en el Bierzo Alto a lo largo del siglo XX.

En primer lugar, y con objeto de conocer la situación de partida a comienzos del siglo $\mathrm{XX}$, se contó como fuente principal con "El Ciclo Vital en España. Encuesta del Ateneo de Madrid, 19011902", del que se extrajo la información relativa a la zona, contenida en el Tomo I, Vol. I y II: Nacimiento. Además, se recurrió también a la consulta de bibliografía de carácter etnográfico, con el fin de constatar y enriquecer con más detalle los hechos (Alonso Ponga y Diéguez Ayerbe, 1984; Caro Baroja, 1977; Casado Lobato, 1992; Díez Alonso, 1982; Fernández Álvarez y Breaux, 1998; García Martínez, García Martínez, Valle Racero, 1994; García Rodríguez, 1996; Juanas Baragaño, Martínez Pérez, y Bachiller Ramón, 1998; Rodríguez y Rodríguez, 1995; Rúa Aller y Rubio Gago, 1986; Rúa Aller y Rubio Gago, 1990) 
En segundo lugar se pretendió elaborar un listado de las personas que asistían a las mujeres en el momento del parto en la zona objeto de estudio en los años cincuenta del pasado siglo, y así mismo analizar cuál era su papel en dicho proceso. Pạra ello se utilizó como fuente escrita el "Anuario General de España Bailly-Baillière-Riera", años 1930 y 1944, Tomo III, un anuario comercial donde se recogía una rica información general de los pueblos: número de habitantes, profesionales o comercios existentes, además de los nombres de los profesionales sanitarios que trabajaban en la subcomarca. Dada la imposibilidad de entrevistar a la comadrona de los años 30 (o en su defecto al practicante) en Bembibre (ambos ya fallecidos), y puesto que tampoco se logró contactar con ninguna de las restantes parteras de la zona, se entrevistó a una matrona que, si bien ejerció como tal en un centro hospitalario de Ponferrada, asistió también a muchos partos en distintas localidades del Bierzo Alto, durante el citado período. Como complemento a sus datos, se añadieron algunas informaciones facilitadas por la sobrina de la comadrona de Bembibre en los años 1930 y 1944.

Por otra parte, y dado que todavía viven un número considerable de mujeres, mayores de 65 años pero que pasaron su juventud en la comarca, entendimos que en este grupo poblacional podía existir una fuente importante de información. Ello permitió plantearse un segundo objetivo: conocer quiénes eran realmente las personas que se dedicaban a atender a la mujer del medio rural durante el parto. Con este fin se realizó un recorrido por distintos pueblos del entorno, preguntando a las mujeres de mayor edad cómo se realizaba la asistencia a la parturienta en sus localidades. Durante este recorrido se conoció a una de estas "mujeres sin título" que se dedicaba "a ayudar a parir", y a la cual se entrevistó.

Finalmente, también se entrevistó a la matrona que trabaja actualmente en el Centro de Salud de Bembibre.

\section{RESULTADOS}

\subsection{LA ASISTENCIA EN EL PARTO A PRINCI-} PIOS DEL SIGLO XX EN EL BIERZO ALTO

La Ley de Instrucción Pública de 9 de Septiembre de 1875 , institucionalizaba la profe- sión de Practicante y Matrona, definiendo a ésta como "mujer práctica en el arte de partos o que ejerce en virtud del título". Eran pues ellas las profesionales encargadas de asistir a los partos normales, y a los de urgencia hasta la llegada del médico. Así lo reflejaba también el "Manual de partos", escrito por Francisco Alonso Rubio en 1866, donde se describían las formas de actuación de las matronas en diversas situaciones anormales. Los requisitos para acceder a estos estudios, impartidos en las Casa de Matronas o en los hospitales con "sala de partos", eran: tener más de 20 años, la primera enseñanza completa, estar casada (en ese caso se precisaba la autorización de su marido) o viuda, y además un certificado de buena conducta expedido por el párroco. En 1888 se aprobó el reglamento para las carreras de Practicante y Matrona, quedando sometida la acreditación de sus conocimientos a jurisdicción médica. En 1902 se autorizó a los practicantes a asistir a los partos normales, lo que generó más de una polémica con las matronas (Siles González, 1995; Siles González, 1996; García Martínez y García Martínez, 1999).

Para conocer cómo y quién prestaba la atención al parto en el Bierzo Alto, a principios del siglo $\mathrm{XX}$, se contó, como se dijo, como principal fuente escrita con las respuestas a la "Encuesta del Ateneo de Madrid, 1901-1902”. Específicamente, en el tercer apartado del Tomo I de dicha encuesta, nos narraban los informantes quién era la mujer encargada de ayudar a la parturienta durante el alumbramiento. Dada su amplitud, se recogen sólo algunas consideraciones de mayor interés. Así, se dice que en poblaciones importantes de la provincia de León, la asistencia al parto correspondía a médicos comadrones y comadronas autorizadas, pertenecientes al sector profesional sanitario; sin embargo, cabe añadir que la zona objeto de estudio estaba formada por núcleos poblacionales de pequeño tamaño, en los cuales eran las parteras (mujeres sin título profesional), las que atendían en casa de la parturienta a la misma; basando su actuación en sus habilidades prácticas tales como frotar al niño con vino blanco "para que tomara fuerza"; así como en las creencias mágico-religiosas y populares transmitidas por la tradición, como por ejemplo: utilizar el cornezuelo del centeno para acelerar las contracciones del parto u otras hierbas; amule- 
tos como la "piedra de rayo", que atada en la pierna izquierda de la parturienta auguraba "un parto rápido"; o la cinta de las embarazadas para evitar que el niño naciera de nalgas o le "entraran males extraños"; encender velas bendecidas el Jueves Santo; rezar oraciones; o aplicar el "bautismo de urgencia" en casos de necesidad.

\subsection{LA ASISTENCIA EN EL PARTO A} MEDIADOS DEL SIGLO XX EN EL BIERZO ALTO

Situados ya en la década de los cincuenta, los datos se obtuvieron mediante un "trabajo de campo", en el que se preguntó a personas veteranas (señoras mayores, pero con buena lucidez mental) de diferentes pueblos, lo que permitió conocer los nombres bastantes mujeres encargadas de ayudar a dar a luz entre los años 1930 y 1970 . Cuando se pudo contactar con alguna de estas parteras o matronas, se realizó una entrevista abierta para conocer cómo era su forma de trabajo y los medios disponibles entonces (por limitaciones de espacio del artículo se comentan sólo los datos más relevantes de las mismas). Además, partiendo de los datos publicados en el "Anuario General de España, Bailly-Baillière-Riera" $(1930,1944)$ y de los datos obtenidos en el referido "trabajo de campo", se elaboró un listado con las matronas y parteras que ejercían entre los años 1930 y 1944 (ANEXO I y II).

Esta investigación permitió contrastar y diferenciar, en la subcomarca que nos ocupa, la labor de las comadronas (que poseían un título oficial), y las curiosas (mujeres sin título, pero con ciertas habilidades para el ejercicio de esta práctica).

En los dos casos se trataba de personal femenino. Ambas figuras ejercían en la misma época y territorio. Según cuentan la comadrona y la curiosa entrevistadas, así como las demás informantes, la comadrona solía atender los partos "de la gente más pudiente" de los pueblos, mientras que las capas más populares eran asistidos por la curiosa.

Según el "Anuario General de España", en 1930 sólo había dos comadronas en el Bierzo Alto, mientras que en 1944 el número de comadronas se había duplicado, ascendiendo a cuatro. Si bien no existe ningún registro documental ni bibliográfico que haga mención al número de curiosas existen- tes; basándonos en los datos facilitados por los informantes de algunos pueblos de la subcomarca, se puede afirmar que en cada localidad solía haber, dependiendo del tamaño de la población, al menos una o dos curiosas.

Todos los informantes comentaron que tanto a las comadronas como a las curiosas se las trataba de "señora...". Era una manera que tenían las gentes de manifestar su respeto y gratitud hacia esas mujeres. La comadrona profesional y la curiosa entrevistadas coinciden en afirmar que sólo las primeras percibían retribución económica por sus servicios. Este hecho viene determinado por el reconocimiento del estatus académico que tenían las comadronas, no en vano poseían un título oficial que les avalaba para ejercer como tales, mientras que las curiosas no.

Por otra parte, durante los meses que duraba la gestación la embarazada no recibía atención alguna de la matrona ni de la curiosa. Su asistencia se limitaba al momento del parto. Bien es cierto que los cuidados de las comadronas se prolongaban un poco más en el tiempo que el de las curiosas. En los días posteriores al nacimiento ellas eran las encargadas de visitar a la madre y velar por su salud y la de la criatura. Aunque tanto comadronas como curiosas dejaban que la madre fuera la verdadera protagonista del parto, interviniendo lo mínimo en el proceso natural. La comadrona entrevistada contó cómo en ciertas ocasiones empleaba alguna medicación u otras técnicas para facilitar el proceso del parto.

La curiosa conocía y llevaba a cabo, por otra parte, más creencias y tradiciones populares, desconocidas por la matrona, como por ejemplo administrar el "caldo de gallina" tras el parto, invocar a santos, o realizar técnicas para prevenir el "mal de ojo", regalando a las mujeres a las que atendía escapularios para que protegieran así a su hijo de tal mal. La comadrona, en cambio, se mostraba sorprendida de la pervivencia de estas creencias y prácticas en ciertas personas, y la única tradición popular que confesó realizar fue el "bautismo de urgencia". La curiosa contaba también como las embarazadas y madres acudían al "curandero" para consultar ciertos problemas de su salud, hecho también desconocido por la comadrona. 
3.3. LA ATENCIÓN DE LA EMBARAZADA A PRINCIPIOS DEL SIGLO XXI EN EL BIERZO ALTO

Como en todas las profesiones, durante el siglo $\mathrm{XX}$ se ha producido una gran evolución en cuanto a formas y medios de trabajo disponibles por las matronas. Actualmente ésta es la profesional de obligada referencia para la mayoría de las mujeres embarazadas del Bierzo Alto. Para conocer la labor que desarrollan se entrevistó a la matrona de la Zona de Salud de Bembibre; sin embargo dadas las limitaciones de espacio se ha incluido sólo una reseña con los datos más destacables de la misma.

Cabe decir que, además de actuar como "consejera" de las embarazadas sobre los asuntos y cuestiones relativos al embarazo y parto, también acuden mujeres en edad de la menopausia, así como otras que deseando tener hijos tardan en conseguirlo. Las embarazadas, una vez que conocen su estado, suelen acudir a consulta cada dos meses. En las distintas visitas les informa sobre la higiene y dieta adecuada que deben llevar, de los cuidados a seguir durante el período de gestación. También les proporciona información sobre cómo prepararse para el parto, pautas, ejercicios de relajación.

Cuando ya se acerca el momento del alumbramiento, la mujer es llevada al centro hospitalario para dar a luz. Allí será otra matrona la que le acompañe y ayude en ese momento. Con la generalización del parto atendido en los centros hospitalarios, se introducen nuevas formas en el protocolo para abordar el mismo. La matrona de Bembibre cuenta cómo a la parturienta se le realizan, casi sistemáticamente, varias técnicas invasivas: la episiotomía, la administración de enemas o la anestesia. Además, durante la expulsión del niño, se le impone la postura ginecológica, sin que le quede otra opción. También la figura del médico está mucho más presente en el parto que antes.

Después de dar a luz y ser dada de alta, la nueva madre visita a los pocos días al Centro de Salud para que la matrona les realice, a ella y a la criatura, un examen de salud, y proporcione a la madre nuevos consejos y pautas sobre el cuidado del recién nacido.

La matrona está pues, desde el primer momento, en contacto con la embarazada, siendo quien la orienta y guía durante el período pre y post parto.
La matrona del Centro de Salud manifiesta no ser conocedora de la mayoría de las creencias y tradiciones populares aún vigentes entre algunas embarazadas.

\section{DISCUSIÓN DE RESULTAdOS Y CON- CLUSIONES}

Basándonos en la "Encuesta del Ateneo, 19011902", en los datos extraídos del "Anuario General de España, Bailly-Baillière-Riera" (1930, 1944), en las entrevistas realizadas a las matronas y curiosas, y en los testimonios aportados por ciertas mujeres mayores, se pueden extraer una serie de conclusiones acerca de algunos de los "cuidados enfermeros" prestados por estas profesionales a lo largo del siglo XX.

A principios del siglo $\mathrm{XX}$, nos encontramos en los pueblos del Bierzo Alto con la figura de la partera o curiosa como encargada de atender los partos. Se trata de una mujer "sin título", pero dotada de cierta habilidad adquirida gracias a la práctica (debido el alto índice de natalidad de entonces, en cualquier pueblo, por pequeño que fuese, existía una o varias), que acude a casa de la parturienta para auxiliarle y "cogerle" a su hijo en ese momento trascendental. No faltan tampoco en su protocolo de actuación la aplicación de creencias y rituales culturalmente aceptados, dada la credibilidad y el respeto que generalmente inspira su persona. En todo caso, no realiza un seguimiento del embarazo; y en cuanto al lugar de alumbramiento se producía éste en el hogar y muchas veces en la propia cocina.

Tal situación se prolonga durante un período de tiempo un tanto impreciso, si bien a partir de la década de los cincuenta cada vez va siendo más frecuente, especialmente en los pueblos más cercanos a Bembibre, la figura de comadrona titulada. Ello se debe a que una hija del pueblo cursó estos estudios, por lo que atendía a las mujeres del entorno que requerían sus servicios, hasta que trasladó su residencia fuera. En otros casos, tratándose de familias con más medios económicos, también se solicitaba la asistencia de alguna comadrona de Ponferrada. La comadrona titulada (sector profesional) por el contra que la curiosa (sector popular) se implica profesionalmente en el cuidado del recién nacido y de la madre en los días posteriores, orientando a ésta en la "cultura de la maternidad". 
A partir del último tercio de siglo, con la progresiva generalización de la atención sanitaria hasta su total universalización, la cultura del embarazo y parto siguieron también parecidas pautas. Actualmente y una vez conocido el estado de gravidez, la mujer se pone en contacto con la matrona o el personal sanitario correspondiente, acudiendo periódicamente a su consulta en el Centro de Salud. Aquí obtiene información y las pautas a seguir según el momento de embarazo, seguimiento del mismo, protocolo del parto, cuidados que debe dar al recién nacido cuando llegue el alumbramiento, higiene, dieta, lactancia. A pesar de que la matrona toma contacto desde los primeros meses con la embarazada y su mundo, conoce poco acerca de las creencias y tradiciones populares (Andina Díaz, en prensa). Además, otro dato importante de las últimas décadas es el hecho ya generalizado de la hospitalización previa al parto, hasta tal punto esto ha pasado a ser algo normal que lo excepcional es el "parto extrahospitalario".

Ya para concluir, se puede afirmar que se han cumplido los objetivos propuestos inicialmente en el trabajo, al igual que la hipótesis planteada al comienzo "la atención en Enfermería durante el embarazo y parto está basada hoy en día en el paradigma biomédico dominante, obviando ese lado mágico y religioso que acompañaba hace un siglo a la atención prestada por las antiguas matronas".

De unos cuidados basados en creencias y tradiciones populares se ha pasado a una atención en Enfermería fundamentada en el conocimiento científico, trasladando el escenario donde se da a luz, siendo una de las premisas básicas la tecnifi- cación y medicalización del parto.

Las ciencias de la salud se ha apropiado en los últimos decenios de funciones que hace un siglo eran patrimonio de la familia, como el embarazo, parto, o crianza de los hijos. Esta nueva forma de abordar el nacimiento, tratándola desde el punto de vista biológico y medicalizando los procesos que acontecen, en la que se propugna una atención enfermera basada en métodos cuantitativos, en un enfoque positivista, y buscando el perfeccionamiento en la tecnología médica y las técnicas como sello de identidad (Rohrbach-Viadas, 1997), deja en un plano casi olvidado la relación con las personas, los valores humanos, en definitiva, otras esferas que también forman parte de la maternidad, el ámbito social y cultural (Balaguer Perigüell y otros, 1991; Martínez Hernáez, 1992; Andina Díaz, en prensa).

Por último, resaltar que dado que los datos con los que se contó fueron parciales y selectivos, no se realizó una investigación exhaustiva. Este hecho, no obstante, no invalida que dichos resultados puedan servir para completar otras investigaciones sobre el tema (Rossi y O’Higgins, 1981). Ello permitiría ahondar aún más, enriqueciendo y contrastando lo ahora publicado.

El rol de la Enfermería, El Cuidado, es Universal; pero el contenido del trabajo de la enfermera es diferente, está modificado por la utilidad o valor cultural particular de cada sociedad.

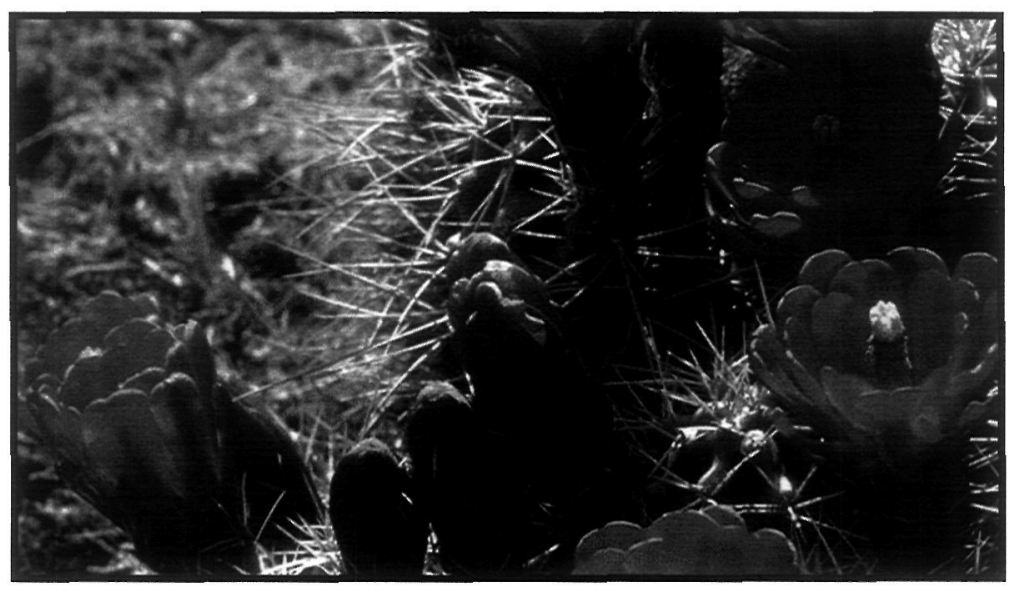




\section{ANEXO I \\ ELENCO DE CURIOSAS DEL BIERZO ALTO, AÑOS CINCUENTA}

Fuente: "informantes" de los pueblos del Bierzo Alto

\section{Municipio: BEMBIBRE}

\section{BEMBIBRE}

\section{CURIOSAS}

- "Señora Lorenza". Años 60. Informante: Sras. del Taller de Costura de Bembibre.

- "Señora Andrea (barrio de La Estación)". Informante: Sras. del Taller de Costura.

- "Señora Silveria (San Román y Bembibre)". Años 60. Informante: Sras. del Taller de Costura.

\section{CURANDERAS}

- "Angel Alonso, barbero y curandero". Informante: María Falagán (nieta), Residencia de la Tercera Edad, Bembibre.

- "Señora Lucía". Falleció hace poco.Informante: Sras. del Taller de Costura.

- "Señora Benita", también "componedora". Actuaba hace unos 60 años. Informante: Sras. del Taller de Costura.

- "Señora Lorenza", que además de partera era curandera. Informante: Sras. del Taller de Costura y M $\mathrm{M}^{\mathrm{a}}$ Ángeles González.

- "Señora ?" que echa las cartas, a lo mejor también se dedica a quitar mal de ojo. Informante: $\mathrm{M}^{\mathrm{a}}$ Ángeles González.

\section{ABORTOS}

- "Señora Leonor". Trabajaba de "clandestina". Informantes: Sras. del Taller de costura.

\section{Municipio: CASTROPODAME}

\section{CASTROPODAME}

\section{CURIOSAS}

- "Sra. Asunción García". Antes de los años 50. Informante: Oliva Reguero.

- "Sra. Isabel Fernández Álvarez". Algo anterior a Dorotea. Informante: Oliva Reguero.

- "Dorotea García García". Cogía niños entre mediados de la década de los 30 hasta 1973 aproximadamente. Informante: Oliva Reguero.

\section{VILLAVERDE DE LOS CESTOS CURIOSAS}

- "Señora Isabel, (la Ferrera)". Años 1930-50. Informante: Ascensión Mauriz, 81 años.

- "Señora Elisa". Años 1945-60. Informante: Ascensión Mauriz, 81 años.

\section{TURIENZO CASTAÑERO CURIOSAS}

- “Señora Rosario Payero". Años 1950. Informante: Amparo Rodríguez Rodríguez.

- "Señora Aurora Fuentes". Años 1950. Informante: Amparo Rodríguez Rodríguez.

\section{Municipio: FOLGOSO DE LA RIBERA}

\section{LA RIBERA DE FOLGOSO CURIOSAS}

- "Señora María Otero". Hasta los 50. Informante: Señora Carmen Alonso.

- "Señora María".- Informante: Señora Carmen Alonso.

\section{TEDEJO}

\section{CURANDERA Y CURIOSA}

- "Señora María, (Tía María)". Año 1946. Informante: Sras. del Taller de Costura y Sra. Engracia.

\section{VILLAVICIOSA DE PERROS CURIOSAS}

- "Señora Felipa". Años 60. Informante: Sras. del Taller de Costura.

\section{Municipio: IGÜEÑA}

\section{IGÜEÑA}

\section{CURIOSAS}

- "Nadie". Solían ayudar las otras mujeres de la familia. Fuente: Ivo.

\section{ALMAGARINOS CURIOSAS}

- "Sra. Dominga (Dominga Marcos)". Años 30 hasta los años 50. Informante: Joaquina Blanco Fernández. 
- "Sra. Balbina García". Atendía hacia 1968. Era un poco mayor que Dominga. Informante: Joaquina Blanco Fernández.

- "Sr. Bernardo (Bernardo García)". Era capador de animales, curandero y en alguna ocasión también actuó como partero. Falleció por los años 60. Informante: Joaquina Blanco Fernández.

\section{POBLADURA DE LAS REGUERAS CURIOSAS}

- "Sra. Antonia". Tiene cerca de 90 años, vive en León. Informante: Florinda García Fdez. y otras.

- "Sra. Antonia (Antonia Iglesias)", vive en el pueblo. Informante: Florinda García Fdez. y otras.

\section{QUINTANA DE FUSEROS CURIOSAS}

- "Sra. Josefa Segura (la Chareta)". Cogía niños allá por los años 40-60. Informante: Pilar Muñiz.

\section{TREMOR DE ARRIBA CURIOSAS}

- "Sra. María (María Blanco)". Tiene unos 90 años y vive en Astorga. Atendía por los años 40 hasta casi los 80. Informante: Gregoria Peña Pozo y Herminia Crespo Blanco.

- "Sra. Elisa (Elisa Raimundo)". "Cogía" por los años 60. Informante: Gregoria Peña Pozo y Herminia Crespo Blanco.

\section{Municipio: NOCEDA}

\section{NOCEDA}

\section{CURIOSAS}

- "Señora Matilde" (Barrio de San Pedro), Por los años 30. Informante: $\mathrm{M}^{\mathrm{a}}$ Ángeles González Arias.

\section{ARLANZA CURIOSAS}

- "Sra. Engracia" (Engracia González Cobos). Años 1950-65. Informante: Sra. Engracia. (Entrevista aparte).

\section{LOSADA}

\section{CURIOSAS}

- "Doña Paquita". Era la Maestra y también actuaba como curiosa. Años 50. Informante: Sra. Engracia.

\section{Municipio: TORRE DEL BIERZO}

\section{TORRE DEL BIERZO CURIOSAS}

- "Señora Gregoria Rivera”. Hasta los años 60. Informante: Felicidad Rivera.

\section{GRANJA DE SAN VICENTE CURIOSAS}

- "Sra. Esperanza (Esperanza Vidal)". Era curiosa, "cogía" niños antes de la Guerra. Informante: Rosalina Silván, 84 años.

- "Sra. Dionisia (Dionisia Fernández)". Antes y después de la Guerra. Informante: Rosalina Silván, 84 años.

- "Sra. Maruja". Años 50 y 60. Informante: Rosalina Silván, 84 años.

\section{SAN ANDRÉS DE LAS PUENTES CURIOSAS}

- "Sra. Nicolasa (Nicolasa García)", ya fallecida, ayudaba a los de la familia. Informantes: Ludivina y Rosa.

- "Sra. Laura". Años 50 y 60. Informantes: Ludivina y Rosa.

\section{SAN FACUNDO \\ CURIOSAS}

- "Sra. Dionisia (Dionisia Blanco Mendaña)". Era partera y "encañadora". Falleció hace más de 42 años. Informantes: Adoración Paz Morán (hija de Isabel Morán), Esther Otero García.

- "Sra. Isabel (Isabel Morán Blanco, hija de Dionisia Blanco)". Falleció en agosto de 1995 con 78 años. "Cogía" niños por la década de los 50 y 60. Informantes: Adoración Paz Morán (hija de Isabel Morán), Esther Otero García.

- "Sra. Antonia". Sólo atendía a sus hijas. La señora Antonia había venido de Matavenero. Informantes: Adoración Paz Morán (hija de Isabel Morán), Esther Otero García.

- "Sra. Isabel (la hija de Antonia)". Informantes: Adoración Paz Morán (hija de Isabel Morán), Esther Otero García. 
- "Sra. Joaquina (Joaquina Álvarez Morán)". Informantes: Adoración Paz Morán (hija de Isabel Morán), Esther Otero García.

- "Sra. Julia (Julia Lodos, era de Villaemil ?, Puentenuevo, Lugo)". Informantes: Adoración Paz Morán (hija de Isabel Morán), Esther Otero García.

- "Sra. Lorenza". Residía en Bembibre. También acudió a asistir algunos partos a $\mathrm{S}$. Facundo. Informantes: Adoración Paz Morán (hija de Isabel Morán), Esther Otero García.

\section{TREMOR DE ABAJO}

\section{CURIOSAS}

- "Sra. Dolores (Dolores Álvarez)". Informante: Cecilia y Oliva.

\section{ANEXO II}

ELENCO DE MATRONAS DEL BIERZO ALTO, AÑOS CINCUENTA

Anuario General de España.Tomo III. 1930 y 1944. Bailly-Baillière-Riera

\section{Municipio: BEMBIBRE}

\section{BEMBIBRE}

COMADRONA

- María Alonso. Año 1930 y 1944.

- Oliva, a partir de los años 50. A.T.S. y Matrona. Informante: María Falagán y Sras. del Taller de Costura.

\section{PRACTICANTE}

- Leoncio Alonso. Año 1930 y 1944.

\section{MÉDICO, GINECÓLOGO Y CIRUJANO}

- Modesto Fernández. Año 1930 y 1944.

\section{Municipio: CASTROPODAME}

\section{CASTROPODAME COMADRONA}

- Manuela Álvarez. Año 1944.

MÉDICO

- Rafael González. Año 1930.

- Isidoro Sarasola. Año 1944.

\section{Municipio: IGÜEÑA}

IGÜEÑA

MÉDICO

- Manuel Estévez. Año 1930.

\section{TREMOR DE ARRIBA}

COMADRONA

- Baltasara Peña. Año 1930.

\section{MÉDICO}

- Bernardo García. Año 1930.

Municipio: NOCEDA

NOCEDA

COMADRONA

- Benita Arias. Año 1944.

MÉDICO

- Ezequiel Polo. Año 1944.

\section{Municipio: TORRE DEL BIERZO}

TORRE DEL BIERZO

MÉDICO:

- Manuel Fernández. Año 1944.

ALBARES DE LA RIVERA (antes era del Ayuntamiento de Torre del Bierzo) COMADRONA

- Francisca Álvarez. En Albares?. Año 1944.

\section{MÉDICO}

- Manuel Fernández. Año 1930.

FOLGOSO DE LA RIVERA (antes era del Ayuntamiento de Torre del Bierzo)

\section{MÉDICO}

- Manuel Estévez. Año 1930.

- Manuel Padilla. Año 1944. 


\section{BIBLIOGRAFÍA}

- Alonso Ponga, J. L., Diéguez Ayerbe, A. (1984), Etnografía y folklore de las comarcas leonesas. El Bierzo, Ed. Leonesas, León.

- Andina Yanes, J. (1977), Estudio socio-económico de Bembibre, (inédito), Bembibre.

- Andina Díaz, E. (en prensa), Un siglo en las creencias y prácticas populares acerca de la gestación y alumbramiento en el Bierzo Alto (León), Index de Enfermería.

- Anuario General de España (1930 y 1944), BaillyBaillière-Riera.

- Balboa de Paz, J. A. (1996), Visita El Bierzo, Everest, León.

- Balaguer Perigüell, E., Ballester Añón, R., Bernabeu Mestre, J., Nolasco Bonmatí, A. Y Perdiguero Gil, E. (1991), Fenómenos biológicos y fenómenos culturales. La interpretación popular del Ciclo Vital en dos comunidades alicantinas. Monografías sanitarias, Serie D, n 10 , Salud para todos en el año 2000, Consellería de Sanitat i Consum. Generalitat Valenciana, Valencia.

- Caro Baroja, J. (1997), Los pueblos del norte, (3 ed.), Txertoa, San Sebastián.

- Casado Lobato, C. (1992), El nacer y el morir en tierras leonesas, Colección Serie León por dentro, Surcos 3, Ed. Caja España, León.

Cuadri Duque, M.J. (1999), Antecedentes históricos de la formación de las matronas, Híades, IV (5-6), págs. 261-270.

- Díez Alonso, M. (1982), Mitos y Leyendas de la Tierra Leonesa, Gráficas León, León.

Fernández Álvarez, D. Y Breaux, J. (1998), Medicina popular, magia y religión en El Bierzo, Museo del Bierzo, Peñalba impresión, Ponferrada.

- García Martínez, M.J. y García Martínez, A.C.(1999), Fechas clave para la historia de las matronas en España, Híades, IV (5-6), págs. 243-260.

- García Martínez, M.J., García Martínez, A.C. y Valle Racero, J.I. (1994), La administración del bautismo de urgencia: una función tradicional de matronas, Matronas hoy, 4, págs. 47-53.

- García Rodríguez, A. (coordinador) [1996], Bierzo mágico, Diario de León, León.

- Juanas Baragaño, J. J., Martínez Pérez, E. y Bachiller Ramón, C. (1998), Revisión antropológica de la maternidad, Matronas hoy, 11, págs. 39-53.

- Limón Delgado, A. y Castellote Herrero, E. (1990), El Ciclo Vital en España. Encuesta del Ateneo de Madrid, 1901-1902. Tomo I. Vol I. Nacimiento, Museo del Pueblo Español, Madrid.

- Littlewood, J. y Holden, P. (1991), Anthropology and Nursing, Routledge, London and New York.

- Martínez Hernáez, A. (1992), Eficacia simbólica, eficacia biológica. Hacia un nuevo modelo analítico y terapéutico en la asistencia sanitaria, Rol de Enfermería, XV (172), págs. 61-67.

- Mulhall, A. (1996), Anthropology, nursing and midwifery: a natural alliance?, International Journal of Nursing Studies, 33 (6), págs. 629-637.

- Rodríguez y Rodríguez, M. (1995), Etnografía folklórica del Bierzo. Oeste-suroeste, Peñalba impresión, Ponferrada.

Rohrbach-Viadas, C. (1997), Cuidar es antiguo como el mundo y tan cultural como la diversidad humana, Cultura de los Cuidados, I (2), págs. 36-39.

- Rossi, I. y O’Higgins, E. (1981), Teorías de la cultura y métodos antropológicos, Anagrama, Barcelona.

- Rúa Aller, F. y Rubio Gago, M.E., (1986), La piedra celeste. Creencias populares leonesas, Imprenta provincial, León.

- Rúa Aller, F. y Rubio Gago, M.E., (1990), La medicina popular en León, Ed. Leonesas, León.

- Siles González, J. (1995), Estructura familiar y función social de la mujer en Alicante: 1868-1936. Consellería de Educación y Ciencia. Generalitat Valenciana, Instituto Juan Gil-Albert, Alicante.

- Siles González, J. (1996), Pasado, presente y futuro de la Enfermería en España, Consejo de Enfermería de la Comunidad Valenciana, Alicante.

- Siles González, J. (2001), Los textos como fuente de conocimientos en Ciencias Sociales y Enfermería, Index de Enfermería, X (34), págs. 33-36.

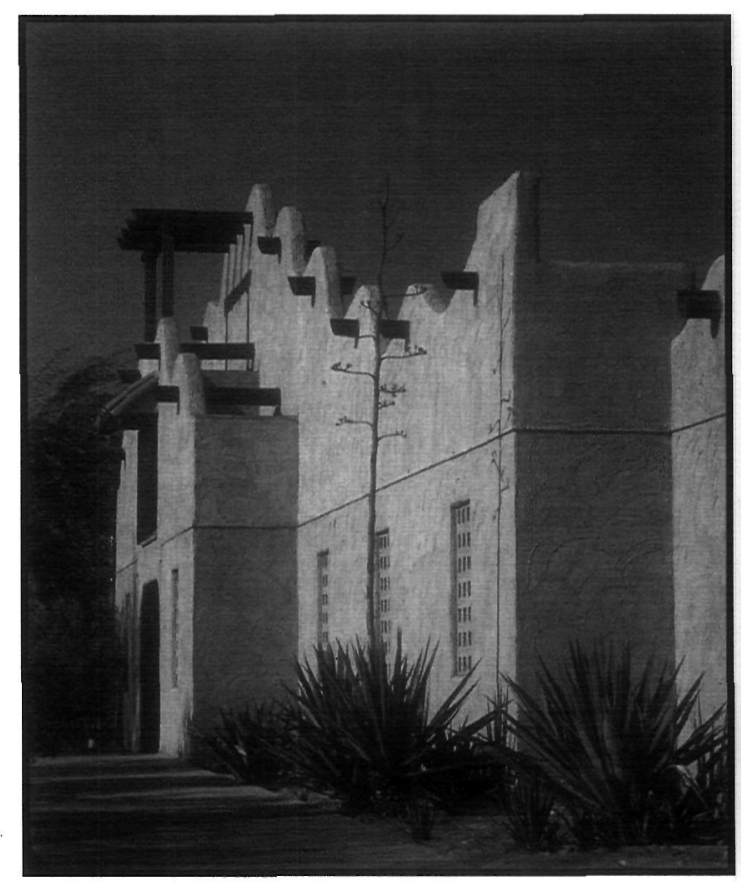

